\title{
Evidências científicas das neurociências para a terapia cognitivo-comportamental
}

\author{
Patrícia Porto \\ Universidade Federal do Rio de Janeiro, Rio de Janeiro-RJ, Brasil \\ Letícia Oliveira \\ Universidade Federal Fluminense, Niterói-RJ, Brasil \\ Eliane Volchan \\ Universidade Federal do Rio de Janeiro, Rio de Janeiro-RJ, Brasil \\ Jair Mari \\ Universidade Federal de São Paulo, São Paulo-SP, Brasil \\ Ivan Figueira \\ Paula Ventura \\ Universidade Federal do Rio de Janeiro, Rio de Janeiro-RJ, Brasil
}

\begin{abstract}
Resumo: Os transtornos de ansiedade estão envolvidos com a incapacidade de controlar o medo e a dificuldade em regular emoções negativas. A terapia cognitivo-comportamental (TCC) abrange técnicas que permitem tanto a extinção do medo condicionado quanto a regulação cognitiva de emoções. Este artigo tem como objetivo apresentar resultados de estudos de regulação da emoção que podem ilustrar a associação e colaboração entre a TCC e as neurociências. Amparado nas pesquisas de regulação da emoção, este estudo corrobora os resultados dos ensaios clínicos com TCC revelando que as intervenções de distração, reestruturação cognitiva e exposição são eficazes para a regulação emocional.
\end{abstract}

Palavras-chave: Terapia cognitivo-comportamental. Neurociências. Ansiedade.

\section{Scientific evidences from neurosciences to cognitive behavioral therapy}

\begin{abstract}
Anxiety disorders are related with inability to control fear and regulate negative emotions. The cognitive behavioral therapy (TCC) encompasses techniques that allow both the extinction of fear conditioning and cognitive emotional regulation. The study aims to report results of emotion regulation studies that can illustrate the association and collaboration between TCC and neuroscience. Based on emotion regulation studies, this study corroborates previous findings of clinical trials with TCC, revealing that interventions like distraction, cognitive re-structuring and exposure are effective in regulating emotions.
\end{abstract}

Keywords: Cognitive behavioral therapy. Neurosciences. Anxiety.

\section{Evidencias científicas de las neurociencias para la terapia cognitiva-conductista}

Resumen: Los trastornos de ansiedad establecen relación con la incapacidad de controlar el miedo y la dificultad en regular emociones negativas. La terapia cognitiva-conductista (TCC) abarca técnicas que permiten tanto la extinción del miedo condicionado, como la regulación cognitiva de emociones. Este artículo tiene como objetivo presentar resultados de estudios de regulación de la emoción que pueden ilustrar la asociación y colaboración entre la TCC y las neurociencias. Anclado en las pesquisas de regulación de la emoción este estudio corrobora los resultados de los ensayos clínicos con la TCC al revelar que las intervenciones de distracción, reestructuración cognitiva y exposición son eficaces para la regulación emocional.

Palabras clave: Terapia cognitiva-conductista. Neurociencias. Ansiedad. 
Os estudos mostram que a terapia cognitivocomportamental (TCC) tem modelos de tratamento em vários transtornos mentais com índices elevados de eficácia (Beck, A. T., 2005; Beck, J. M., 2007; Foa, 2006). Esta terapia oferece uma perspectiva interessante para a integração com o campo da neurociência, uma vez que qualquer intervenção está vinculada a um suporte de pesquisa experimental e empírico (De Raedt, 2006).

O intercâmbio da TCC com a neurociência é o diálogo entre mente e cérebro. Podemos considerar que mente e cérebro são integrados e interdependentes. Os processos mentais exercem influência na plasticidade cerebral em vários níveis como celular, molecular e em circuitos neurais (Beauregard, 2007; Kumari, 2006). Para ilustrar essa relação Beauregard cita que pensamentos que induzem medo aumentam a secreção de adrenalina, enquanto pensamentos relacionados à felicidade aumentam a secreção de endorfina. Os processos neurais estão envolvidos com outros processos fisiológicos como o imune e o endócrino que por sua vez estão associados à comunicação entre os processos mentais e cerebrais. De fato, parafraseando Paqquette e cols. (2003) "mudando sua mente você pode modificar seu cérebro".

As pesquisas em neurociências podem colaborar para potencializar nosso conhecimento sobre as bases neurobiológicas das psicopatologias e da TCC, assim como auxiliar no refinamento de intervenções a fim de aumentar a eficácia do tratamento. Os estudos de regulação de emoção são exemplos de como podemos integrar os achados da neurociência às teorias da TCC. Com o intuito de incitar a reflexão sobre esse tema, o presente artigo tem como objetivo apresentar resultados de estudos de regulação da emoção, especificamente a ansiedade, que podem ilustrar a associação e colaboração entre esses dois campos do conhecimento: a TCC e as neurociências.

\section{Contribuições das neurociências para a terapia cognitivo-comportamental}

Os estudos mostram que muitos transtornos mentais estão envolvidos com a incapacidade de controlar o medo (LeDoux, 1998; Liggan \& Kay, 1999) e dificuldade em regular emoções negativas (Mocaiber e cols., 2008; Ochsner \& Gross, 2005). Esses dados sugerem que o condicionamento de medo e a dificuldade em regular emoções têm importante papel na formação e manutenção especialmente dos transtornos de ansiedade. Mocaiber e cols. ressaltam que a pesquisa sobre os circuitos neurais da extinção tem importante implicação clínica. Isto porque os transtornos de ansiedade são em parte caracterizados pela resistência à extinção de reações emocionais aprendidas a estímulos ansiogênicos e por comportamentos de evitação.

O tratamento com TCC abrange técnicas específicas que permitem tanto a extinção do medo condicionado quanto a regulação cognitiva de emoções, ou seja, abrange técnicas comportamentais e cognitivas, que serão citadas mais detalhadamente ao longo deste trabalho.

\section{Bases biológicas da ansiedade}

Pesquisas em neurociências têm revelado que a amígdala apresenta-se como estrutura importante no processo de medo condicionado. Quando estamos na presença de perigo ocorrem reações endócrinas, autonômicas e comportamentais em nosso organismo. A ativação da amígdala produz a estimulação de redes responsáveis pelo controle da expressão de uma variedade de reações (Charney, 2003; LeDoux, 1998; Sotres-Bayon, Cain, \& LeDoux, 2006).

A amígdala compõe-se de vários núcleos e nem todos estão implicados no condicionamento do medo, mas os núcleos central e lateral têm participação fundamental neste processo. O núcleo central constitui a principal conexão com as áreas que controlam as reações emocionais (Charney, 2003; LeDoux, 1998; Sotres-Bayon e cols., 2006).

O cérebro é programado para detectar perigos, tanto aqueles com que nossos ancestrais se defrontavam rotineiramente quanto aqueles aprendidos atualmente por cada um de nós, a amígdala e suas conexões de entrada e saída são centrais nestes processos. Estudos mostram que homens e animais adquirem medo de objetos que são potencialmente perigosos ao longo do tempo. A capacidade de reconhecer e responder rapidamente a um estímulo potencialmente perigoso é vantajosa para a sobrevivência. Por conseguinte, 
seríamos preparados biologicamente para responder a estímulos perigosos de modo a garantir a preservação de nossa espécie. Isto é notório ao perceber que alguns indivíduos têm fobias específicas, por exemplo, por ofídios, que atualmente são menos ameaçadores para os seres humanos se comparados a outros estímulos, como armas de fogo. Dessa forma, podemos produzir respostas de proteção mais eficientes para o nosso biotipo e expressar as reações que foram selecionadas para as condições ambientais ancestrais (Hofmann, 2008; LeDoux 1998). Os estímulos que foram evolutivamente selecionados são detectados rapidamente no ambiente. Tal fato sugere que eles capturam a atenção a fim de serem percebidos automaticamente. De modo geral, os indivíduos mostram-se sensíveis à detecção destes estímulos, o que se acentua quando o estímulo realmente provoca medo, isto é, o indivíduo tem fobia a ele (Öhman, 2005). Em síntese, todos nós temos percepção aguçada dos estímulos potencialmente perigosos e essa sensibilidade é maior nas pessoas com fobias a eles.

Os sistemas anatômicos relacionados ao aprendizado do medo permitem respostas rápidas aos estímulos percebidos como potencialmente perigosos e também respostas mais demoradas envolvidas com processamento mais elevado da informação de estímulos sensoriais complexos e o contexto ambiental. As respostas mais rápidas estão relacionadas às projeções do tálamo sensorial para amígdala lateral. Essa projeção envolve o condicionamento rápido para características auditivas e visuais simples responsáveis por respostas de medo de baixo nível de consciência. Já as respostas mais demoradas envolvem projeções do córtex sensorial de associação e estruturas corticais mesotemporais para a amígdala. Essa projeção está relacionada a respostas condicionadas para estímulos sensoriais mais complexos (Charney, 2003). O trajeto de informações tálamo-amígdala é mais curto e mais rápido, mas conforme citado, passa ao largo do córtex e não pode fazer uso de um processamento mais elaborado. Como resultado, fornece apenas uma representação grosseira do estímulo à amígdala. Esta rápida via direta permite-nos dar início à reação diante de um estímulo potencialmente perigoso, antes mesmo de termos plena consciência de qual se trata. Assim, o processamento cortical não é necessário para a informação alcançar a amígdala devido à via direta do tálamo, o reconhecimento consciente de um estímulo aversivo não é necessário para ativar respostas de medo (LeDoux, 1998; Öhman, 2005), o que pode ser muito útil em situações de perigo. No entanto, essa vantagem exige que o caminho cortical seja capaz de ignorar a via direta em situações que não representem perigo real. Assim, uma das funções do córtex seria impedir reações inadequadas (LeDoux, 1998). Do ponto de vista da sobrevivência, é mais interessante reagir ao perigo em potencial como se fosse real do que deixar de fazê-lo, as vias subcorticais fornecem uma imagem grosseira do mundo, enquanto as representações mais detalhadas e precisas provêm do córtex.

As vias tálamo-amígdala e córtex-amígdala convergem no núcleo lateral da amígdala que parece ter um papel importante na coordenação dos processos sensoriais envolvidos na resposta de medo condicionado. Depois que a informação sensorial passa pela amígdala lateral, a representação neural do estímulo é distribuída em paralelo para vários núcleos da amígdala que podem ser modulados por sistemas como o de memórias de experiências passadas ou relacionados ao estado homeostático do indivíduo e deflagram todo um repertório de reações de defesa (Charney, 2003; LeDoux, 1998). Portanto, quando ocorre uma situação desagradável pode ocorrer o condicionamento de medo e assim esses estímulos serão reconhecidos antes mesmo do indivíduo ter consciência deles. Outras estruturas além da amígdala também estão envolvidas no condicionamento do medo como: córtex mesotemporal, tálamo, córtex sensorial, córtex préfrontal orbital e medial, ínsula anterior, hipotálamo, hipocampo e múltiplos núcleos do tronco cerebral (Charney, 2003).

\section{Técnicas comportamentais e os estudos de regulação da emoção}

O tema principal dos pensamentos automáticos dos pacientes ansiosos relaciona-se ao perigo. $\mathrm{O}$ processamento cognitivo nos transtornos de ansiedade envolve a vulnerabilidade, isso porque os pacientes tendem a superestimar o perigo e a subestimar os recursos pessoais para lidar com as situações que são interpretadas como perigosa, a partir do momento 
em que ocorre uma avaliação de perigo ficam propensos a desenvolver mais pensamentos que mantêm a ansiedade. Quando os esquemas cognitivos relacionados ao perigo são ativados, as avaliações caracterizam-se por pensamentos automáticos negativos relacionados a catástrofes físicas, psicológicas ou sociais. Os próprios sintomas de ansiedade decorrentes de uma avaliação de perigo podem transformar-se em nova ameaça percebida, uma vez que prejudicam o desempenho ou são considerados como possível sinal de ameaça (Barlow, 2002; Falcone, 2001; Hofmann, 2008; Leahy, 2006).

Durante o tratamento, o paciente é encorajado a desafiar esses pensamentos, reavaliando sua expectativa de perigo. A exposição é uma das estratégias utilizadas com essa finalidade. Durante as exposições o paciente fortalece seu senso de controle reduzindo expectativas futuras de dano e aumentando seu senso de auto-eficácia. A exposição favorece o teste da realidade e por meio da constatação real de que as conseqüências catastróficas não vão ocorrer, o indivíduo apresenta redução da ansiedade e deixa de emitir as respostas evitação. Tendo como referência essas informações, Hofmann (2008) propõe que a estratégia de exposição para ser conduzida com sucesso requer mudanças nesses processos cognitivos. Assim, a exposição seria uma forma de intervenção cognitiva que promove mudanças na expectativa de perigo dos pacientes ansiosos. Dessa forma, Hofmann caracteriza-a não como uma técnica comportamental, mas cognitiva, mesmo que ela não utilize estratégias de reestruturação cognitiva explícitas, tem efeito similar por mediar mudanças nos pensamentos disfuncionais.

Izquierdo (2004) enfatiza que a técnica de exposição utilizada pela TCC incita o paciente com fobia à "arte de esquecer", uma vez que promove extinção do medo condicionado, auxiliando na remissão dos sintomas. O processo de extinção tem importante valor adaptativo, visto que possibilita a não realização de comportamentos ou respostas que seriam inadequadas. No entanto, a extinção não se deve à perda de um aprendizado, mas sim à formação de um novo que se superpõe ao anterior e que inibe a resposta deste. A recuperação espontânea, que se caracteriza pela pronta recuperação da resposta de medo que já havia sido extinta, fortalece essa hipótese, sugerindo que a extinção não apaga a memória que associa as reações de medo aos estímulos, mas impede que os estímulos deflagrem a reação. A memória do medo, uma vez estabelecida, se torna relativamente permanente, sendo assim, a extinção possibilita o controle da reação de medo, em lugar da eliminação da memória emocional por si só (Hermans, Craske, Mineka, \& Lovibond, 2006; Izquierdo, 2004; LeDoux, 1998; Quirk, Garcia, \& González-Lima, 2006; Sotres-Bayon e cols., 2006). Assim, a terapia através da técnica de exposição colabora para a ativação de estruturas cerebrais responsáveis pela redução da expressão de medo.

A extinção do medo envolve interação entre áreas cerebrais corticais e subcorticais, especialmente a interação entre o córtex pré-frontal e amígdala (Sotres-Bayon e cols., 2006; Hermans e cols., 2006; Quirk e cols., 2006). Após a extinção, a habilidade de o estímulo condicionado controlar a resposta condicionada através da comunicação entre o núcleo lateral da amígdala e o núcleo central é regulada pelo córtex pré-frontal medial (Sotres-Bayon e cols.). As projeções recíprocas entre a amígdala e o córtex pré-frontal medial possivelmente representam um papel crucial na atenuação de respostas de medo e na extinção de respostas comportamentais de medo condicionado (Charney, 2003). A atividade entre essas regiões também pode ser modulada pela informação contextual gerada pelo hipocampo (Sotres-Bayon e cols.). Conseqüentemente, evidências convergem para a participação especial destas três regiões cerebrais na extinção do medo condicionado: o córtex pré-frontal ventromedial, amígdala e hipocampo, sobretudo a influência do córtex pré-frontal medial regulando a expressão do medo e inibindo a amígdala.

A ansiedade não está somente associada ao aumento da atividade da amígdala, mas também a menor eficiência de modulação do pré-frontal sob esta região. O sistema subcortical e o córtex pré-frontal influenciam-se reciprocamente para modular a resposta emocional que seria mais adequada para lidar com as circunstâncias (Öhman, 2005).

É necessário ressaltar que a técnica de exposição quando associada a sinais de segurança tem se mostrado menos eficaz do que quando os pacientes 
são encorajados a focalizar em seus medos. A presença do terapeuta e a possibilidade de evitar a situação deflagradora de ansiedade são exemplos de sinais de segurança (Hermans e cols., 2006; Rachman, Radomsky, \& Shafran, 2008). Por conseguinte, as respostas de evitação compartilham as mesmas propriedades que os sinais de segurança interferindo nos benefícios da técnica de exposição. Essas condições influenciam o processo de extinção e acabam afetando a mudança da resposta comportamental, apresentando efeito deletério sobre o progresso da exposição. O psicólogo deve orientar o paciente ansioso a abster-se dos comportamentos de evitação a fim de promover redução significativa do medo após a sessão de exposição. É necessário ressaltar que expor o paciente ao estímulo fóbico utilizando tais sinais de segurança e comportamentos evitativos não favorecerá a extinção do medo condicionado. Entretanto, o uso judicioso dos sinais de segurança, especialmente nos estágios iniciais, pode ser um facilitador da técnica de exposição além de reduzir os altos níveis de recusas e abandono da terapia. Cabe ao psicólogo ficar atento ao momento em que a continuidade dos comportamentos de segurança pode interferir com a eficácia do tratamento (Rachman e cols.).

A influência do medo contextual nos sintomas de ansiedade é outro aspecto importante para a eficácia da técnica de exposição. LeDoux (1998) ressalta que durante o condicionamento do medo o indivíduo está com sua atenção voltada tanto para o estímulo mais relevante quanto para estímulos ambientais. Assim, o condicionamento pelo medo contextual compõe-se de todos os estímulos presentes no ambiente e não apenas do estímulo condicionado explícito.

Os estudos em neurociência revelam que as projeções do hipocampo para amígdala via fórnix estão implicadas especificamente no condicionamento contextual espacial (Charney, 2003). LeDoux (1998) aponta que experimentos com ratos que possuem lesões de hipocampo eliminaram seletivamente as reações de medo produzidas pelos estímulos do contexto, mas não influenciaram as reações deflagradas pelo estímulo específico condicionado. Uma das funções do hipocampo é criar uma representação do contexto que contenha não apenas estímulos individuais, mas sim as relações entre os estímulos. O hipocampo analisa e armazena informações relativas ao espaço (LeDoux). O córtex perihinal rostral e córtex pré-frontal ventromedial e ínsula anterior (agranular) também estão envolvidos com a modulação do medo contextual (Charney).

Mudanças de contexto, assim como sinais de segurança, levam à recuperação de respostas aparentemente extintas, quando o paciente se expõe a contextos novos que não foram trabalhados durante o tratamento pode sentir ansiedade. Contextos associados ao tratamento de exposição bem sucedido ativam memórias não relacionadas ao medo, enquanto contextos que não participaram do tratamento podem eliciar memórias de respostas de medo e retorno do mesmo (Hermans e cols., 2006). Conseqüentemente, o psicólogo deve facilitar a ocorrência de exposições que abranjam diferentes contextos a fim de obter melhores resultados. No entanto, nem sempre é possível realizar as exposições em múltiplos contextos ou no ambiente em que o medo foi adquirido. Uma forma de minimizar esse fato seria utilizar estratégias cognitivas em novos contextos para neutralizar o medo que possivelmente surgisse. Como exemplos de estratégias cognitivas que podem favorecer a extinção do medo contextual, citam-se: lembrar do terapeuta no contexto em que ocorreu a extinção do medo e lembrar de informações relacionadas ao tratamento (Hermans e cols.). O medo contextual está relacionado a uma coleção de estímulos e não a um estímulo específico, aspecto que deve ser trabalhado no processo terapêutico.

\section{Técnicas cognitivas e os estudos de regulação da emoção}

Pacientes ansiosos tendem a focalizar em estados corporais que são compreendidos como aversivos, ou seja, interpretam sensações interoceptivas como perigosas ou ameaçadoras. Paulus e Stein (2006) propõem que essa tendência é mediada pelo do circuito neural que tem a ínsula anterior como parte muito importante. Os autores apontam que indivíduos com propensão à ansiedade apresentam alteração na predição de sinais interoceptivos, isto significa que os pacientes ansiosos experimentam um erro na diferença entre o estado corporal observado e o esperado. 
Embora a maioria dos estudos sobre ansiedade focalize o papel da amígdala, Paulus e Stein (2006) ressaltam a participação da ínsula nos transtornos de ansiedade. Segundo esses autores, há vários motivos para considerar que as funções da ínsula juntamente com a amígdala fazem parte da etiologia da ansiedade. O córtex insular está envolvido com a geração de sinais antecipatórios que são importantes para o aprendizado de respostas aversivas. Como o córtex insular faz parte do sistema interoceptivo pode prover informações sobre o estado corporal aversivo futuro, ou seja, sobre as respostas corporais futuras associadas com o estímulo condicionado. Essas informações são enviadas para áreas cerebrais críticas para alocação da atenção e de ações executivas, como o cingulado anterior.

A interocepção pode ser definida como o senso de condição fisiológica de todo o corpo. A informação interoceptiva inclui sensações como: temperatura, dor, dentre outras, e tem sido apontada como parte importante para a auto-consciência porque proporciona uma associação entre processos cognitivos, afetivos e o estado do corpo. A informação sobre o estado interoceptivo processado na ínsula anterior é retransmitida para o córtex cingulado anterior, que faz parte do sistema executivo central responsável pela alocação dos recursos atencionais disponíveis (Paulus \& Stein, 2006).

Nagai, Kishi e Kato (2007) em seu estudo de revisão mostram que a ínsula está envolvida no processamento da raiva, medo, felicidade, repugnância e outros estímulos emocionais aversivos. Estes autores apontam que o córtex insular pode estar envolvido com transtornos de humor, pânico, estresse pós-traumático, obsessivo-compulsivo, compulsão alimentar e esquizofrenia. Etkin e Wager (2007) em sua metanálise de estruturas relacionadas aos transtornos de fobia específica, social, e transtorno de estresse pós-traumático encontraram uma via comum para ansiedade: a hiperativação da amígdala e também da insula.

Tendo como base a teoria de Paulus e Stein (2006), poderíamos ressaltar vários motivos para incluir a ínsula na etiologia da ansiedade. $O$ primeiro deles se refere às conexões anatômicas entre o córtex insular, límbico e áreas do funcionamento executivo importantes na alteração das sensações fisiológicas homeostáticas e na avaliação cognitiva que ocorre em indivíduos com ansiedade ao manifestar hiperestimulação autonômica e preocupação. Outro fator seria o foco na alteração da predição de sinais, que é consistente com os quadros de ansiedade que envolvem cognições e emoções orientados para o futuro. $\mathrm{O}$ organismo tende a prever uma série de alterações associadas aos pensamentos e emoções relacionados às previsões futuras por ele estabelecidas.

Podemos mencionar a teoria cognitivocomportamental para o desenvolvimento do transtorno de pânico a fim de exemplificar a relação entre medo das sensações corporais e ansiedade. Os modelos teóricos enfatizam o papel da reação de medo das sensações físicas na manutenção do transtorno de pânico (Rangé \& Bernik, 2001; Ventura, 2005). Podemos, assim, perceber que os pacientes com pânico tendem a focalizar muito a atenção nas sensações físicas por eles experimentadas. A focalização da atenção nas sensações somáticas levaria a um aumento da ansiedade e consequientemente aumentaria as sensações físicas, favorecendo a focalização ainda maior da atenção nas sensações corporais. Durante o tratamento com TCC uma das estratégias empregada é a técnica de distração, que tem como objetivo incentivar o paciente a focalizar a atenção em outros estímulos, que não as sensações físicas, auxiliando na redução dos sintomas de ansiedade (Rangé \& Bernik; Ventura).

A hipótese da participação da ínsula predizendo estímulos corporais ameaçadores e transmitindo essas informações para o córtex cingulado anterior, que aloca os recursos atencionais disponíveis para esses estímulos, é compatível com a teoria cognitivocomportamental. Essa hipótese também serve de embasamento científico para a estratégia de distração utilizada como uma das intervenções no tratamento do transtorno de pânico, sendo importante ressaltar que outros circuitos neurais também nele estão envolvidos. A matéria cinzenta periaquedutal, na sua porção dorsal tem sido apontada como estrutura importante para a origem do ataque de pânico (Brandão, Zanoveli, Ruiz-Martinez, Oliveira, \& Landeira-Fernandez, 2008). 
Mocaiber e cols. (2008) corroboram com a hipótese anterior ao sugerir em seu estudo que os transtornos de ansiedade podem ser parcialmente explicados pela facilidade em engajar ou desengajar a atenção nos estímulos ou situações negativas. A estratégia de distração é citada como um modo de regulação emocional. Dessa forma, os estudos de regulação da emoção fortalecem a proposta terapêutica da TCC de que a diminuição da alocação dos recursos atencionais nos estímulos emocionais pode reduzir o impacto deste no indivíduo (Mocaiber e cols., 2008; Ochsner \& Gross, 2005).

A importância da atenção para a percepção está fundamentada na capacidade limitada do indivíduo perceber uma situação. Assim, o potencial de uma tarefa relevante exaurir recursos de processamento irá determinar se estímulos distratores serão percebidos ou não. Conseqüentemente, quando o indivíduo tem sua atenção focalizada em um evento relevante pode falhar na detecção de outros estímulos concorrentes, fenômeno conhecido como cegueira atencional (Mocaiber e cols., 2008). Assim, cabe destacar que a percepção e o processamento de um estímulo pode não ocorrer devido ao esgotamento de recursos atencionais na tarefa principal. No entanto, certos estímulos emocionais podem ser percebidos em decorrência do processamento automático que independe da alocação voluntária da atenção, conforme citado anteriormente. Alguns estímulos emocionais possuem seu processamento cerebral privilegiado e capturam preferencialmente a atenção. A necessidade de detecção rápida de estímulos potencialmente perigosos revelam o valor adaptativo de tal processamento (Mocaiber e cols.). Entretanto, em indivíduos com transtornos psiquiátricos no qual um estímulo é compreendido erroneamente como perigoso, este processamento pode gerar prejuízos no dia-a-dia.

Mocaiber e cols. (2008) enfatizam que, embora certos estímulos possam ser percebidos automaticamente, quando o indivíduo desempenha uma tarefa considerada muito difícil que exige conseqüentemente mais recursos cerebrais, o processamento efetivo do estímulo emocional seria dificultado. Desta forma, os estímulos emocionais podem ter seu processamento cerebral diminuído em situações nas quais há pouco recurso atencional disponível. Esse achado é de grande importância para a eficácia do processo terapêutico de pacientes ansiosos. Conseqüentemente, ao longo do tratamento, a técnica de distração para ser eficaz deve priorizar atividades do cotidiano do paciente que reduzam a disponibilidade atencional para estímulos aversivos, a fim de reduzir sua ansiedade.

Erk, Abler e Walter (2006) em seu estudo apontam que existe um continuum de estratégias de regulação da emoção que se estendem desde o controle atencional até a reavaliação cognitiva que permitem modular a percepção emocional e respostas emocionais. Logo, a regulação cognitiva da emoção se dá por meio do controle atencional ou por mudanças cognitivas mais elaboradas (Erk e cols., 2006; Mocaiber e cols., 2008; Ochsner \& Gross, 2005).

A mudança cognitiva pode ser utilizada para regular uma resposta emocional que já foi iniciada através da reavaliação (Ochsner \& Gross, 2005). A reavaliação cognitiva está relacionada à regulação da emoção por meio da capacidade de interpretar situações emocionais de forma a limitar a resposta emocional subseqüente. Essa regulação possibilita a redução da experiência negativa, favorecendo a diminuição da ativação simpática (Mocaiber e cols., 2008; Ochsner \& Gross). Assim, a reestruturação cognitiva utilizada na TCC pode ser considerada como uma estratégia de regulação da emoção, uma vez que modula as emoções por meio de mecanismos cognitivos reduzindo o alerta fisiológico. Conseqüentemente, quando um paciente ansioso consegue reestruturar seus pensamentos disfuncionais, ou seja, interpretar a situação de forma mais adaptativa, ele estaria reduzindo seu grau de ansiedade através de mecanismos cognitivos (Mocaiber e cols.).

Quanto aos circuitos neurais, a estratégia de reavaliação cognitiva se mostra relacionada à atividade do cingulado anterior dorsal (Ochsner \& Gross, 2005) e do córtex pré-frontal (Mocaiber e cols., 2008; Ochsner \& Gross) associada à diminuição da ativação da amígdala (Mocaiber e cols.). Possivelmente o córtex pré-frontal estaria modulando a atividade da amígdala. Esses achados são compatíveis com os circuitos envolvidos com o medo condicionado, especialmente, córtex pré-frontal medial e amígdala, mencionados anteriormente. 
Erk e cols. (2006) apontam que a reavaliação cognitiva e a técnica de distração são eficazes para redução de uma emoção negativa antecipatória. No entanto, quando o indivíduo utiliza apenas estratégia de distração, ela não impede que a antecipação negativa volte a ocorrer. Neste sentido, a distração cognitiva é uma estratégia com efeitos pontuais, atua no momento da antecipação reduzindo a resposta emocional, mas não tem eficácia a longo-prazo, no sentido de evitar a antecipação negativa. Tal fato se deve à distração não ser voltada para a modificação da percepção de um evento emocional que é antecipado, isto é, não tem orientação para futuro. A utilização de uma estratégia mais orientada para o futuro, objetivando modificar a resposta emocional através de uma reavaliação do evento, produzirá efeitos maiores sobre a antecipação emocional. Conseqüentemente, a técnica de distração é eficiente para atenuar a resposta emocional durante a antecipação, mas não para a redução antecipatória aversiva por si (Erk e cols.). Esses achados justificam porque diferentes técnicas da TCC são aplicadas para eficácia do tratamento em pacientes ansiosos. Tendo como referência tais resultados a reestruturação cognitiva mostra-se mais interessante para reduzir a ansiedade antecipatória dos pacientes, uma vez que esta técnica favorece a busca de evidências a fim de avaliar se os pensamentos são compatíveis com a realidade do indivíduo. A falta ou insuficiência de evidências que comprovam ou não os pensamentos possibilitam a reavaliação da situação, promovendo alteração na emoção do paciente.

\section{Considerações finais}

Podemos integrar as pesquisas em neurociências às psicoterapias (Etkin, Pittenger, Polan, \& Kandel, 2005; Moras, 2006). Os estudos de regulação de emoção apresentados neste trabalho foram exemplos dessa colaboração para a TCC.

A pesquisa sobre os circuitos neurais dos transtornos mentais tem importante implicação clínica, pois amplia nosso conhecimento dos mecanismos neurobiológicos subjacentes às patologias (Liggan \& Kay, 1999), assim como podem revelar os circuitos neurais associados à melhora dos sintomas em decorrência do tratamento bem sucedido com TCC (Beauregard, 2007; Etkin e cols., 2005; Kumari, 2006; Linden, 2006; Porto, 2007; Roffman, Marci, Glick, Dougherty, \& Rauch, 2005). Conseqüentemente, estes estudos podem contribuir para o aumento da eficácia da terapia quer através do aperfeiçoamento de novas técnicas quer através da potencialização com fármacos. Em síntese, a compreensão das bases neurobiológicas relacionadas ao processo emocional é de grande importância para a psicologia.

Os estudos mostram que os transtornos de ansiedade são em parte caracterizados pela resistência à extinção de reações de medo, pelos comportamentos de evitação (LeDoux, 1998; Liggan \& Kay, 1999) e pela dificuldade em regular emoções negativas (Mocaiber e cols., 2008; Ochsner \& Gross, 2005). Os estudos de regulação da emoção mencionados neste trabalho evidenciam que as estratégias de exposição, distração e reestruturação cognitiva são eficazes para a regulação emocional. Assim, é importante ressaltar que o tratamento com TCC abrange técnicas específicas que permitem tanto a extinção do medo condicionado quanto a regulação cognitiva das emoções. Os resultados dos estudos de regulação da emoção descritos neste trabalho fortalecem a proposta terapêutica da TCC, revelando ser esta uma modalidade de psicoterapia amparada tanto em evidências científicas de estudos de ensaios clínicos quanto das ciências básicas.

\section{Referências}

Barlow, D. H. (2002). Anxiety and its disorders: The nature and treatment of anxiety and panic (2nd ed.). NewYork: Guildford Press.

Beauregard, M. (2007). Mind does really matter: Evidence from neuroimaging studies of emotional self-regulation, psychotherapy, and placebo effect. Progress in Neurobiology, 81(4), 218-236.

Beck, A. T. (2005). The current state of cognitive therapy: A 40-year retrospective. Archives General Psychiatry, 62, 953-959.

Beck, J. S. (2007). Terapia cognitiva para desafios clínicos: $O$ que fazer quando o básico não funciona. Porto Alegre: Artmed. 
Brandão, M. L., Zanoveli, J. M., Ruiz-Martinez, R. C., Oliveira, L. C., \& Landeira-Fernandez, J. (2008). Different patterns of freezing behavior organized in the periaqueductal gray of rats: Association with different types of anxiety. Behavioral and Brain Research, 188, 1-13.

Charney, D. (2003). Neuroanatomical circuits modulating fear and anxiety behaviors. Acta Psychiatry Scandinavica Supplement, 108(Suppl. 417), 38-50.

De Raedt, R. (2006). Does neuroscience hold promise for the further development of behavior therapy? The case of emotional change after exposure in anxiety and depression. Scandinavian Journal of Psychology, 47, 225-236.

Etkin, A., \& Wagner, T. D. (2007). Functional neuroimaging of anxienty: A meta-analysis of emotional processing in PTSD, social anxiety disorder, and specific phobia. American Journal Psychiatry, 164, 1476-1488.

Etkin, A., Pittenger, C., Polan, H. J., \& Kandel, E. R. (2005). Toward a neurobiology of psychotherapy: Basic science and clinical applications. Journal Neuropsychiatry Clinical Neuroscience, 17, 145-158.

Erk, S., Abler, B., \& Walter, H. (2006). Cognitive modulation of emotion anticipation. European Journal of Neuroscience, 24, 1227-1236.

Falcone, E. (2001). Psicoterapia cognitiva. In B. Rangé (Org.), Psicoterapias cognitivocomportamental: Um diálogo com a psiquiatria (pp. 49-61). Porto Alegre: Artmed.

Foa, E. B. (2006). Psychosocial therapy for posttraumatic stress disorder. Journal of Clinical Psychiatry, 67(Suppl. 2), 40-45.

Hermans, D., Craske, M. G., Mineka, S., \& Lovibond, P. F. (2006). Extinction in human fear conditioning. Biology Psychiatry, 60, 361-368.

Hofmann, S. G. (2008). Cognitive processes during fear acquisition and extinction in animals and humans: Implications for exposure therapy of anxiety disorders. Clinical Psychology Review, 28, 199-210.
Izquierdo, I. (2004). A arte de esquecer: Cérebro, memória e esquecimento. Rio de Janeiro: Vieira e Lent.

Kumari, V. (2006). Do psychotherapies produce neurobiological effects? Acta Neuropsychiatrica, 18, 61-70.

Leahy, R. L. (2006). Técnicas de terapia cognitiva: Manual do terapeuta. Porto Alegre: Artmed.

LeDoux, J. E. (1998). O cérebro emocional: Os misteriosos alicerces da vida emocional. Rio de Janeiro: Objetiva.

Linden, D. E. (2006). How psychotherapy changes the brain: The contribution of functional neuroimaging. Molecular Psychiatry, 11, 528-538.

Liggan, D. Y., \& Kay, J. (1999). Some neurobiological aspects of psychotherapy: A review. Journal Psychotherapy Practice and Research, 8, 103-114.

Mocaiber, I., Oliveira, L., Pereira, M. G., MachadoPinheiro, W., Ventura, P. R., Figueira, I. V., \& Volchan, E. (2008). Neurobiologia da regulação emocional: Implicações para a terapia cognitivocomportamental. Psicologia em Estudo, 13, 531-538.

Moras, K. (2006). The value of neuroscience strategies to accelerate progress in psychological treatment research. Canadian Journal of Psychiatry, 51, 810-822.

Nagai, M. K., Kishi, K., \& Kato, S. (2007). Insular cortex and neuropsychiatric disorders: A review of recent literature. European Psychiatry, 22, 387-394.

Ochsner, K. N., \& Gross, J. J. (2005). The cognitive control of emotion. Trends in Cognitive Sciences, 9, 242-249.

Öhman, A. (2005). The role of the amygdala in human fear: Automatic detection of threat. Psychoneuroendocrinology, 30, 953-958.

Paqquette, V., Lévesque, J., Mensour, B., Leroux, J.M., Beaudoin, G., Bourgouin, P., \& Beauregard, M. (2003). Change the mind and you change the brain: Effects of cognitive-behavioral therapy on the neural correlates of spider phobia. Neuroimage, 18, 401-409. 
Paulus, M. P., \& Stein, M. B. (2006). An insular view of anxiety. Biology Psychiatry, 60, 383-387.

Porto, P. (2007). Estudo de revisão sistemática de terapia cognitivo-comportamental nos transtornos de ansiedade. Dissertação de mestrado não-publicada, Universidade Federal do Rio de Janeiro, Instituto de Psicologia, Rio de Janeiro, RJ.

Quirk, G. J., Garcia, R., \& González-Lima, F. (2006). Prefrontal mechanisms in extinction of conditioned fear. Biology Psychiatry, 60, 337-343.

Rangé, B., \& Bernik, M. (2001). Transtorno de pânico e agorafobia. In B. Rangé (Org.), Psicoterapias cognitivo-comportamentais: Um diálogo com a psiquiatria (pp. 145-182). Porto Alegre: Artmed.

Rachman, S., Radomsky, A. S., \& Shafran, R. (2008). Safety behaviour: A reconsideration. Behaviour Research and Therapy, 46, 163-173.

Roffman, J. L., Marci, C. D., Glick, D. M., Dougherty, D. D., \& Rauch, S. L. (2005). Neuroimaging and functional neuroanatomy of psychotherapy. Psychological Medicine, 35, 1385-1398.

Sotres-Bayon, F., Cain, C. K., \& LeDoux, J. E. (2006). Brain mechanisms of fear extinction: Historical perspectives on the contribution of prefrontal cortex. Biology Psychiatry, 60, 329-336.

Ventura, P. R. (2005). Psicoterapia cognitivocomportamental. In A. E. Nardi \& A. M. Valença (Orgs.), Transtorno de pânico: Diagnóstico e tratamento (pp. 90-98). Rio de Janeiro: Guanabara Koogan.

Artigo recebido em 17/06/2008.

Aceito para publicação em 16/12/2008.

Endereço para correspondência:

Profa. Dra. Paula Ventura. Universidade Federal do Rio de Janeiro. Instituto de Psicologia. Av. Pasteur, 250. CEP: 22290-240. Rio de Janeiro-RJ, Brasil. E-mail: paularventura@uol.com.br
Patrícia Porto é Mestre pelo Programa de Pós-graduação em Psicologia do Instituto de Psicologia da Universidade Federal do Rio de Janeiro.

Letícia Oliveira é Professora Adjunta do Instituto Biomédico e do Departamento de Psiquiatria e Saúde Mental da Universidade Federal Fluminense.

Eliane Volchan é Professora Adjunta do Instituto de Biofísica da Universidade Federal do Rio de Janeiro.

Jair Mari é Professor Titular do Departamento de Psiquiatria da Universidade Federal de São Paulo.

Ivan Figueira é Professor Adjunto do Departamento de Psiquiatria e Saúde Mental da Faculdade de Medicina da Universidade Federal do Rio de Janeiro.

Paula Ventura é Professora Adjunta do Instituto de Psicologia da Universidade Federal do Rio de Janeiro. 